\title{
Dissipative Toda-Rayleigh lattice and its oscillatory modes
}

\author{
V. A. Makarov, ${ }^{1}$ E. del Río, ${ }^{2}$ W. Ebeling, ${ }^{1,3}$ and M. G. Velarde ${ }^{1}$ \\ ${ }^{1}$ Instituto Pluridisciplinar, Universidad Complutense, Paseo Juan XXIII, 1, Madrid 28040, Spain \\ ${ }^{2}$ ETSI Aeronauticos, Universidad Politecnica de Madrid, Plaza Cardenal Cisneros, 3, Madrid 28040, Spain \\ ${ }^{3}$ Institute of Physics, Humboldt-Universität Berlin, Invalidenstrasse, 110, Berlin 10178, Germany \\ (Received 3 October 2000; revised manuscript received 23 May 2001; published 14 August 2001)
}

A detailed theoretical and experimental analysis of the possible oscillatory regimes of the dissipative TodaRayleigh lattice system is provided. It is shown that the system has $(N-1)$ oscillatory modes with different space-time scales and two rotatory modes. Using its analog electronic circuit implementation we also show with a simple and robust method how switching between modes occurs.

DOI: 10.1103/PhysRevE.64.036601 PACS number(s): 46.05.+b, 05.45.-a, 84.30. $-\mathrm{r}, 45.50 .-\mathrm{j}$

\section{INTRODUCTION}

Recent theoretical [1-17] and experimental [18-30] works dealing with nearly integrable systems and nonlinear dissipative waves have illustrated the lasting structure of solitonic properties during time evolution, in collisions and wall reflections. The theoretical work refers mostly to hydrodynamics, electronics, and optical fiber experiments and, from the theoretical side, to generalizations of the Boussinesq and Korteweg-de Vries equations which are the continuous counterpart of various lattice systems [31,32]. As already discussed by $\mathrm{Chu}$ and Velarde [5] an energy pumping-dissipation balance is the crucial element in a driven system. If the energy is appropriately in balance with dissipation, the soliton originated in an e.g., nonlinearitydispersion (local) balance can be sustained. Besides, the energy balance selects a single phase wave velocity among the infinitely many existing in the one-parameter family of solitary waves of, say, the standard Korteweg-de Vries equation [1,6-12]. A similar selection occurs for periodic wave trains but then we have a two-parameter problem. Here we shall restrict consideration to a dissipation-modified lattice system [33].

Toda [34] provided exact solutions, including solitons, for a Hamiltonian lattice system with exponential interaction. Subsequently, other (conservative) soliton bearing systems were proposed for nonlinear signal processing and transmission. However, observation of waves in nature and engineering devices shows that dissipation is unavoidable and may, on occasion, drastically affect the expected phenomena. Experiments with analog electrical circuit implementations of the Toda lattice and numerical tests have shown that, in practical terms, unavoidable dissipative effects lead to energy losses and indeed spoil the conservative nature of the Toda lattice [35-39]. Thus interest has arisen in studying the role of dissipation and controlled energy pumping, e.g., in the stationary wave propagation along the Toda lattice. Accordingly we study, both analytically and experimentally, a Toda lattice with a Rayleigh-type energy pumping-dissipation balance [40] much in the spirit of the above mentioned works on continuous systems.

The set of equations describing the motions in a chain of point masses coupled by Toda springs including dissipative effects is

$$
\begin{gathered}
\frac{d Y_{n}}{d t}=P_{n}, \\
\frac{d P_{n}}{d t}=\frac{\Omega^{2}}{B}\left(\exp \left[B\left(Y_{n-1}-Y_{n}\right)\right]\right. \\
\left.-\exp \left[B\left(Y_{n}-Y_{n+1}\right)\right]\right)+G\left(P_{n}\right),
\end{gathered}
$$

where $P_{n}, Y_{n}, \Omega$, and $B$ account, in Toda's original problem, for momenta, displacements, base frequency of linear oscillations, and the stiffness of the springs (assumed to be identical). An interesting feature of the Toda interaction is that under appropriate limits it goes into the harmonic oscillator limit and into the hard sphere interaction in another. The nonlinear function $G(P)$ in the system Eq. (1) is going to account for dissipation and sources of energy, which in general can be distributed nonhomogeneously on lattice sites [41].

The system Eq. (1) is chosen with left-right symmetry. This means that if there is a solution $\left(Y_{n}(t), P_{n}(t)\right)$ (a clockwise propagating wave) then there is a solution $\left(-Y_{-n}(t)\right.$, $-P_{-n}(t)$ ) (a counterclockwise propagating wave). Hence we require that $G(-P)=-G(P)$. The simplest nonlinear function satisfying this condition is a cubic polynomial

$$
G(P)=d\left(\widetilde{P}^{2}-P^{2}\right) P,
$$

where $\widetilde{P}$ is a constant having the dimension of momentum. Such cubic nonlinearity describes a pumping-saturation balance introduced by Rayleigh [40] and is widely used in various fields of science [42-45].

Introducing new space and time scales, and corresponding dimensionless variables, $y_{n}=B Y_{n}, p_{n}=(d / B) P_{n}$, we get from Eq. (1)

$$
\begin{gathered}
\dot{y}_{n}=p_{n}, \\
\dot{p}_{n}=\omega_{0}^{2}\left(e^{y_{n-1}-y_{n}}-e^{y_{n}-y_{n+1}}\right)+\left(\mu-p_{n}^{2}\right) p_{n},
\end{gathered}
$$

with

$$
\mu=\frac{\widetilde{P}^{2}}{B^{2}}, \quad \omega_{0}=\frac{d \Omega}{B^{2}}, \quad t_{\text {new }}=\frac{B^{2}}{d} t_{\text {old }} .
$$




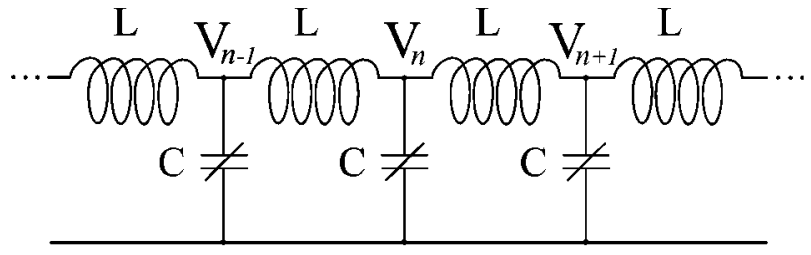

(a)

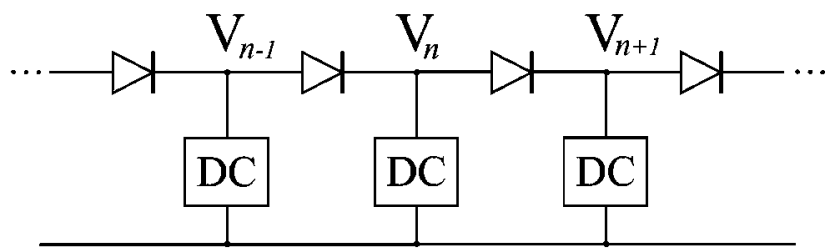

(b)

FIG. 1. Circuit implementations of the Toda lattice: (a) nonlinear $L C$ circuit by Hirota and Suzuki [35], (b) diode-double capacitor circuit by Singer and Oppenheim [36].

The lattice system Eq. (3) has two independent parameters: (i) $\omega_{0}$ which is the base frequency of linear oscillations in the original Toda lattice [34], and (ii) $\mu$ which is the linear pumping constant accounting for the dissipative Rayleigh part of the system. Their values and relation define the spatio-temporal dynamics of the chain. Further, for convenience, we shall use nearest-neighbor relative coordinates along the lattice, i.e.

$$
x_{n}=y_{n-1}-y_{n}
$$

with periodic boundary conditions that demand $\sum_{n=1}^{N} x_{n}=0$.

In contrast to the original Toda equations [34], the system Eq. (3), in a closed ring chain, does not permit the simultaneous propagation of stationary waves traveling in opposite directions [33]. We shall focus on the stable oscillatory modes occurring in a relatively short Toda-Rayleigh ring chain Eq. (3). In Sec. II we give a succinct account of analog circuit implementations of the Toda and Toda-Rayleigh systems. Section III is devoted to the analysis of a single unit. In Sec. IV we investigate the possible wave modes in the ring. Finally, in Sec. V some concluding remarks are given.

\section{ANALOG CIRCUIT IMPLEMENTATION OF THE MODEL}

Let us discuss two different electric circuit models of the Toda lattice that guided us in our analog electrical circuit implementation of the Toda-Rayleigh lattice.

\section{A. Various electrical circuit analogs of the Toda lattice}

For the original Toda lattice, $G(P) \equiv 0$, Hirota and Suzuki [35] have shown that the mechanical chain with exponential interactions is equivalent to a ladder of $L C$ circuits [Fig. 1(a)] with linear inductors and suitable nonlinear capacitors

$$
Q_{n}=C^{0} V^{0} \ln \left(1+\frac{V_{n}}{V^{0}}\right), \quad C(V) \equiv \frac{d Q}{d V}=\frac{C^{0} V^{0}}{V^{0}+V},
$$

with $Q_{n}$ and $V_{n}$ accounting, respectively, for the charge and the voltage at the $n$th capacitor. $C(V)$ is the capacitance. Then the dynamic equations describing the ladder are

$$
\begin{gathered}
\frac{d Q}{d t}=C^{0} V^{0} \frac{d}{d t} \ln \left(1+\frac{V_{n}}{V^{0}}\right)=I_{n+1}-I_{n}, \\
L \frac{d I_{n}}{d t}=V_{n}-V_{n+1},
\end{gathered}
$$

which, indeed, are equivalent to the original Toda system [34]. To approximate the nonlinearity Eq. (5) in the experiments, Hirota and Suzuki used varactor diodes. However, for high voltages the deviation of the varactor diode capacitance from the required one becomes significant. It leads to distortions of the soliton solutions and their collision leads to wavy forms which are self-destructive. Besides, since the circuit is accurate for low voltages only, it is very difficult to observe overtaking soliton collisions.

Recently, Singer and Oppenheim [36] proposed a different electrical circuit supposed to better model the Toda lattice. They used junction diodes which have current-voltage $(I-V)$ characteristics very close to exponential. Hence it is natural to use a number of series connected diodes to model the effect of Toda springs. To compute time derivatives, they used double capacitors [Fig. 1(b)]. The current through a double capacitor is proportional to the second derivative of the voltage. Thus we have

$$
\frac{d^{2} V_{n}}{d t^{2}}=D I_{\mathrm{dc}}=D\left(I_{n-1}-I_{n}\right),
$$

where $I_{\mathrm{dc}}$ is the current through the double capacitor and $D$ is a constant with dimension $\left(\mathrm{V} / \mathrm{s}^{2} \mathrm{~A}\right)$, which depends on internal components of the double capacitor. The current $I_{\mathrm{dc}}$ is equivalent to the difference of currents through neighboring junction diodes $\left(I_{n-1}-I_{n}\right)$, which can be accurately modeled as a function of the voltage applied to the terminals, hence

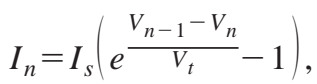

where $I_{s}$ is the saturation current of diodes, and $V_{t}$ is a constant having voltage dimension. Equations (7) and (8) are equivalent to the Toda system [34].

In the Singer-Oppenheim circuit voltage fronts can propagate to the right or to the left depending on the sign of the spatial difference between voltages from the opposite side of the front. Singer and Oppenheim were able to observe "true overtaking soliton collisions with a small number of nodes" [36]. They proposed using such circuits for nonlinear signal processing.

\section{B. Circuit implementation of the Toda-Rayleigh lattice}

Before we proceed further let us note that in general an excitation passing through a unit in the chain increases (or decreases) its voltage by a value depending on the type of 
excitation. For example, for the solitary wave solution of the Toda chain the difference between voltages, before and after the soliton passes a unit, is [36]

$$
\Delta V=\int_{-\infty}^{\infty} p(t) \mathrm{d} t=\kappa V_{t},
$$

where $\kappa$ is an arbitrary constant defining the spatial width of the soliton. Thus a wave rotating in the ring continuously increases (respectively, decreases for a wave running in the opposite direction) the voltage in the units and, eventually, saturates the circuit. To avoid this problem, Singer and Oppenheim [36] used analog switches, allowing us to reset the circuit after each run, which resulted in short spikes in diode currents. These switches are synchronized by an external circuit with an initial pulse imposed by a current generator acting on the first unit of the chain. Here we take the SingerOppenheim electronic implementation of the circuit since it models the Toda equations better than the Hirota-Suzuki circuit. We add a specific implementation of $G(P)$ in Eq. (1) to the circuit and propose a different solution to the saturation problem. Our circuit does not contain switches and any additional synchronization block, i.e., it could work at all times without the need of resetting the capacitors.

The voltages $V_{n}$ on the units can be decomposed into two parts: (i) one always increasing (which leads to saturation), and (ii) another oscillating around some mean value

$$
V_{n}=c t+V_{n}^{\text {new }},
$$

where $c$ is the voltage growth rate. In the mechanical analog (a ring of point masses coupled by springs) the coordinate change defined by Eq. (9) corresponds to a transformation into a reference frame rotating with constant velocity $c$, and thus a wave appears as vibrations of masses around their corresponding fixed mean values. The transformation Eq. (9) does not affect the original "Toda" part, but it leads to a shift in the "Rayleigh" part, $G(d V / d t) \rightarrow G[(d V / d t)+c]$, of the system Eq. (1). In general, for different wave regimes the constant $c$ may be different. Hence we have to introduce an additional electric block to tune the constant $c$ thus eliminating saturation of the circuit.

The Singer-Oppenheim circuit was developed for modeling the original, conservative, Toda system [34]. Consequently, all observed waves come from chosen initial conditions imposed by the current generator. For a dissipative system like Eq. (1) the initial conditions lose significance as we have indicated that the input-dissipation energy balance helps to select and sustain a specific solution, and hence only a limited and well-defined number of different stable solutions are actually realized. Thus we chose between different solutions by playing with an external voltage as we explain below.

The circuit scheme for the Toda-Rayleigh chain is shown in Fig. 2. Figure 3 shows a particular implementation of the black boxes used in Fig. 2. According to the $I-V$ relation of the double capacitor, as in Eq. (7), we have

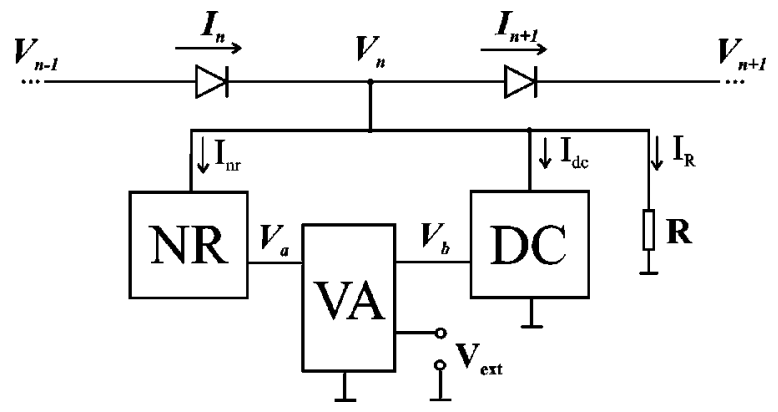

FIG. 2. Block scheme of the Toda-Rayleigh lattice: (DC) is a double capacitor, (NR) accounts for the nonlinear dissipative function $G(P)$ in the model Eq. (1); (VA) provides variable shift of the nonlinear function $G(P)$ to compensate voltage drift; $R$ is an additional stabilizing high value resistor $(R=120 \mathrm{k} \Omega)$. Diodes are 1 n4148.

$$
\frac{d^{2} V_{n}}{d t^{2}}=D I_{\mathrm{dc}}=D\left(I_{n}-I_{n+1}-I_{\mathrm{nr}}-I_{R}\right),
$$

with

$$
D=\omega_{v}^{2} R_{\mathrm{dc}}, \quad \omega_{v}^{2}=\frac{1}{C_{1} R_{1} C_{2} R_{2}},
$$

where $I_{R}$ and $I_{\mathrm{nr}}$ are currents through the corresponding linear $R$ and nonlinear, (NR) resistors (Fig. 2).

The current through the nonlinear resistor (block NR in Fig. 2) $I_{\mathrm{nr}}$ is a nonlinear function of the voltage applied to its terminals, $\Delta V=V_{n}-V_{a}$ (Fig. 2). The voltage-current relation for the nonlinear resistor $I_{\mathrm{nr}}(\Delta V)$, is shown in Fig. 4. The block VA in Fig. 2 is a typical voltage adder. This is used to obtain the linear combination $V_{a}=V_{b}+V_{\text {ext }}$, and hence to shift the variable in the function $I_{\text {nr }}(\Delta V)$ according to the external voltage $V_{\text {ext }}$ which is, simultaneously, applied to all elements in the ring. Finally, the voltage $V_{b}$ at the left side pin of the double capacitor (Fig. 2) is

$$
V_{b}=V_{n}-\frac{1}{\omega_{v}^{2} R_{\mathrm{dc}} C_{1}} \frac{d V_{n}}{d t} .
$$

Thus the argument of the function $I_{\mathrm{nr}}(\Delta V)$ is

$$
\Delta V=\frac{1}{\omega_{v}^{2} R_{\mathrm{dc}} C_{1}} \frac{d V_{n}}{d t}-V_{\mathrm{ext}}
$$

and hence depends on the time derivative of $V_{n}$ and common external voltage $V_{\text {ext }}$.

The term $I_{R}$ on the right-hand side (r.h.s.) of Eq. (10) comes from the large $(120 \mathrm{k} \Omega)$ value of $R$ (Fig. 2). This resistor compensates unavoidable differences between units and prevents saturation of the circuit due to small disturbances. We shall further discuss its role in Sec. IV.

Thus, from Eq. (10) we have 


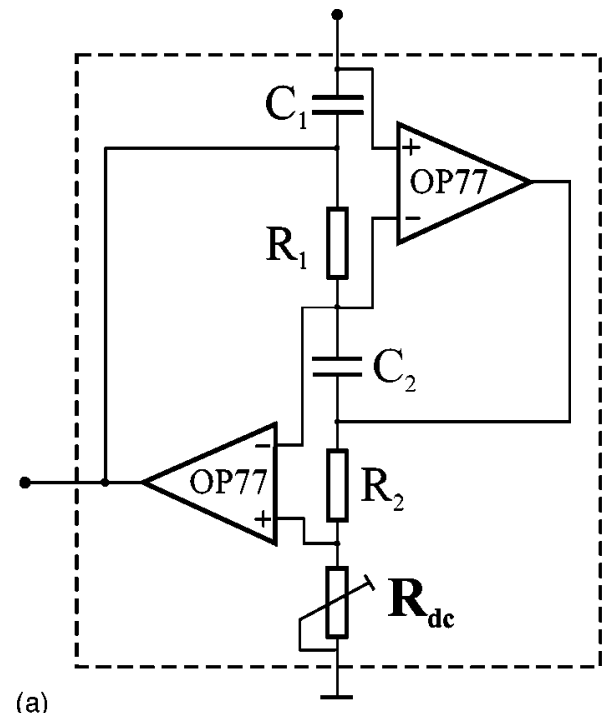

(a)

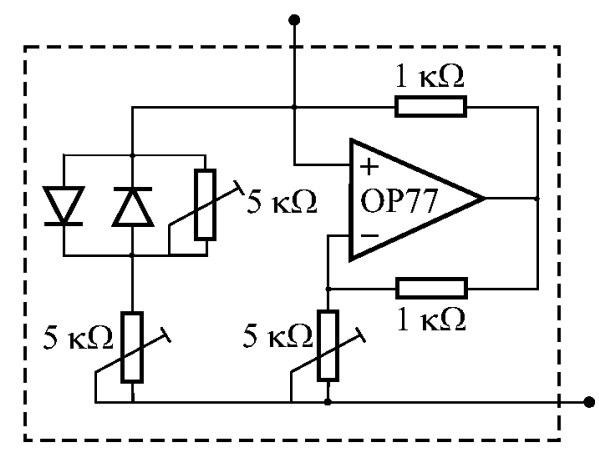

(b)

(c)

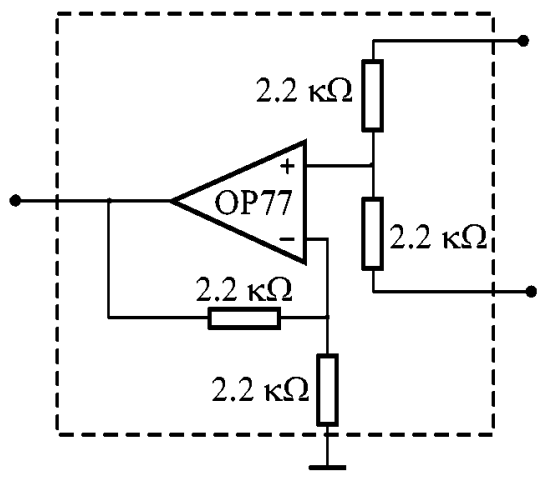

FIG. 3. Particular circuit implementation of the black boxes in the scheme shown in Fig. 2: (a) double capacitor, (b) nonlinear resistor, and (c) voltage adder. Diodes are 1 n4148. Unless otherwise specified we take the following parameter values $C_{1}=C_{2}$ $=0.1 \mu \mathrm{F}, R_{1}=R_{2}=10 \mathrm{k} \Omega$.

$$
\begin{aligned}
\frac{d^{2} V_{n}}{d t^{2}}= & \omega_{v}^{2} R_{\mathrm{dc}}\left\{I_{s}\left[\exp \left(\frac{V_{n-1}-V_{n}}{V_{t}}\right)-\exp \left(\frac{V_{n}-V_{n+1}}{V_{t}}\right)\right]\right. \\
& \left.+I_{\mathrm{nr}}\left(\frac{d V_{n}}{d t}, V_{\mathrm{ext}}\right)-\frac{1}{R} V_{n}\right\} .
\end{aligned}
$$

The direct measurement of $d V_{n} / d t$ (which corresponds to the momentum in the mechanical analog) is not possible. The alternative is to obtain these values from voltage differences

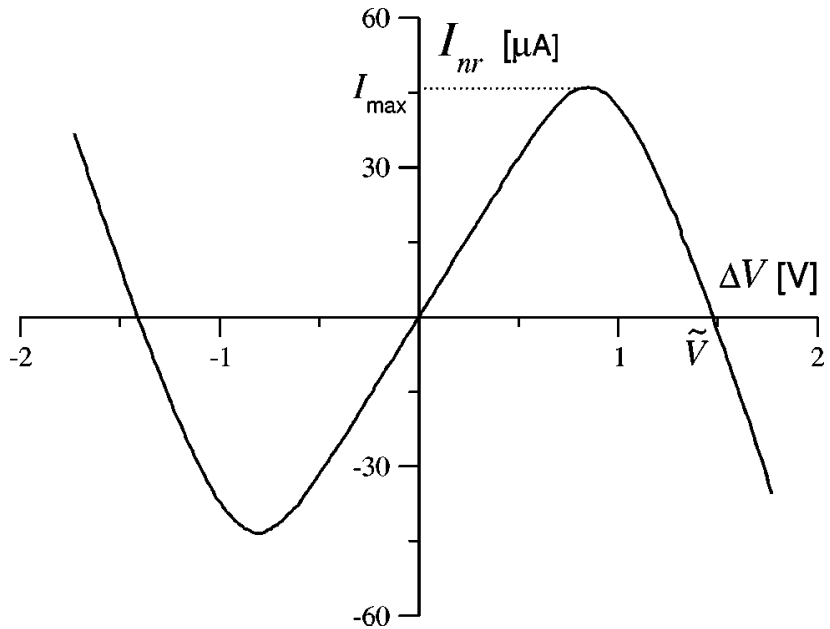

FIG. 4. Experimental current-voltage relation for the nonlinear resistor, $I_{\mathrm{nr}}(\Delta V)$, where $\Delta V=V_{n}-V_{b}-V_{\text {ext }}$ is voltage applied to its terminals.

in a unit using Eq. (11). The function $I_{\mathrm{nr}}$ (Fig. 4) in Eq. (13) plays a similar role to that played by the function $G(P)$ in the system Eq. (1). We can approximate it by a cubic polynomial as in Eq. (2)

$$
I_{\mathrm{nr}}(V)=\frac{3 \sqrt{3} I_{\max }}{2 \widetilde{V}^{3}}\left(\widetilde{V}^{2}-V^{2}\right) V,
$$

where $I_{\max }$ and $\widetilde{V}$ are, respectively, the maximum and zero of the function $I_{\mathrm{nr}}$ (Fig. 4). In our experiments we use $\widetilde{V}$ $\approx 1.45 \mathrm{~V}$ and $I_{\max } \approx 45 \mu \mathrm{A}$.

Comparing the mechanical Eq. (1) and electrical Eq. (13) implementations of the Toda-Rayleigh lattice we get the following relations between parameters and variables:

$$
\begin{gathered}
Y_{n} \rightarrow V_{n}, \quad P_{n} \rightarrow \frac{d V_{n}}{d t}, \quad B \rightarrow \frac{1}{V_{t}}, \quad \Omega^{2} \rightarrow \omega_{v}^{2} \frac{R_{\mathrm{dc}} I_{s}}{V_{t}}, \\
G\left(P_{n}\right) \rightarrow \omega_{v}^{2} R_{\mathrm{dc}} I_{\mathrm{nr}}\left(\frac{1}{\omega_{v}^{2} R_{\mathrm{dc}} C_{1}} \frac{d V}{d t}\right) .
\end{gathered}
$$

Introducing in Eq. (13) new dimensionless variables, $p_{n}$ $=(d / B) P_{n}, y_{n}=B Y_{n}$, with

$$
d=\frac{3 \sqrt{3} I_{\max }}{2 \widetilde{V}^{3} C_{1}^{3} \omega_{v}^{4} R_{\mathrm{dc}}^{2}}
$$

we get a system similar to Eq. (3)

$$
\begin{gathered}
\dot{y}_{n}=p_{n}, \\
\dot{p}_{n}=\omega_{0}^{2}\left(e^{\left.y_{n-1}-y_{n}-e^{y_{n}-y_{n+1}}\right)+\left(\mu-p_{n}^{2}\right) p_{n}-\omega_{R}^{2} y_{n},}\right.
\end{gathered}
$$

with 


$$
\begin{gathered}
\mu=\frac{27 I_{\max }^{2} V_{t}^{2}}{4 R_{\mathrm{dc}}^{2}\left[\widetilde{V} C_{1} \omega_{v}\right]^{4}}, \quad \omega_{0}^{2}=\frac{27 I_{\max }^{2} V_{t}^{3} I_{s}}{4 R_{\mathrm{dc}}^{3}\left[\widetilde{V} C_{1} \omega_{v}\right]^{6}}, \\
\omega_{R}^{2}=\frac{27 I_{\max }^{2} V_{t}^{4}}{4 R R_{\mathrm{dc}}^{3}\left[\widetilde{V} C_{1} \omega_{v}\right]^{6}} .
\end{gathered}
$$

Equation (16) yields Eq. (3) as $R \rightarrow \infty$. For the value of $R$ used in our experiments there is no significant difference in the actual dynamic behavior of these equations.

In Eq. (16), the parameters $\mu, \omega_{0}$, and $\omega_{R}$ depend on $V_{t}$ and, in addition, $\omega_{0}$ depends on $I_{s}$. The experimental problem to evaluate the parameter values of a diode is due to the fact that both $V_{t}$ and $I_{s}$ are strongly temperature dependent. At $300 \mathrm{~K}$ the relative change in $I_{s}$ is about $15 \%$ per $1 \mathrm{~K}$. On the other hand, $I_{s}$ depends strongly on the specific voltage range of the diode operation [46]. In the low voltage range the approximate value of $I_{s}$ is much higher than its value in the high voltage range. Our circuit works in the whole voltage range (from -0.6 to $0.6 \mathrm{~V}$ ). Thus to model the behavior of the real diode we fit the experimental $I-V$ characteristics with Eq. (8) in this voltage range. We get the following values $I_{s} \approx 1.5 \mathrm{nA}$ and $V_{t} \approx 0.05 \mathrm{~V}$. Note that the experimentally obtained value of $V_{t}$ is higher than the thermal voltage $(0.026 \mathrm{~V}$ at $300 \mathrm{~K})$ of the ideal diode. Fortunately, as we shall see in the next sections, it is possible to verify quantitatively the theoretical predictions by using appropriate variables in the circuit that do not depend on $I_{s}$ and $V_{t}$. Their values are important to estimate the relation between the dimensionless parameters $\mu$ and $\omega_{0}$ only. This problem is common for all types of diodes, including the varactors used by Hirota and Suzuki. Finally, all measurements and traces were done by using the digital oscilloscope Yokogawa DL1540C. Traces were digitalized and then plotted with standard computer-graphics tools.

\section{DYNAMICS OF A SINGLE UNIT}

Let us first consider the dynamics of an isolated unit. To do this we connect to ground the terminals of two neighboring diodes. For such configuration the resistor $R$ (Fig. 2) does not play a significant role, since the current through it is negligible relative to the current that flows through two diodes grounded in opposite directions. The configuration just described corresponds to the following boundary conditions for Eq. (3):

$$
y_{n+1}=y_{n-1}=0 .
$$

Hence the evolution of an isolated unit obeys the equation

$$
\ddot{y}+2 \omega_{0}^{2} \sinh y=\mu \dot{y}-\dot{y}^{3} .
$$

When $\sinh y \approx y$, Eq. (17) is the equation first introduced by Rayleigh to describe sustained oscillations in acoustic systems [40].

Omitting the r.h.s., Eq. (17) is a conservative nonlinear oscillator with Hamiltonian $H=\dot{y}^{2} / 2+2 \omega_{0}^{2}(\cosh y-1)$. It has a steady state (a center) at the origin and all other trajectories are closed orbits around it. For $\mu \leqslant 0$, Eq. (17) has a single attractor which is the steady state at the origin. For positive $\mu$ this steady state becomes an unstable node or focus and a single periodic solution that attracts all nonequilibrium trajectories appears through a Hopf bifurcation. Introducing new variables and time scale, $v=-\left(2 \omega_{0}^{2} / \mu^{3 / 2}\right) \sinh y, u$ $=(1 / \sqrt{\mu}) \dot{y}, t_{\text {new }}=\sqrt{\mu} t_{\text {old }}$, Eq. (17) can be rewritten in the form

$$
\begin{gathered}
\dot{u}=\sqrt{\mu}\left(u-u^{3}+v\right), \\
\dot{v}=-u \sqrt{\frac{4 \omega_{0}^{4}}{\mu^{3}}+v^{2}} .
\end{gathered}
$$

By constructing a map of the semiaxis $v^{+}=\{(u, v) \mid v>0, u$ $=0\}$ into itself in a spirit like it was done in Ref. [47] for the Van der Pol equation, one can prove that Eq. (18) and, consequently, Eq. (17) has a single stable limit cycle existing for arbitrary $\mu>0$.

To compare with experimental results, information about the amplitude, period, and shape of the oscillations is needed. Estimate of values of the parameters $\mu$ and $\omega_{0}$ used in the experimental setup shows that $\mu \gg \omega_{0}$. In this limit, changing the time scale $t_{\text {new }}=\left(2 \omega_{0}^{2} / \mu\right) t_{\text {old }}$ and introducing a new variable $v=\left(2 \omega_{0}^{2} / \mu^{3 / 2}\right) y$ we get from Eq. (17)

$$
\begin{gathered}
\dot{v}=u, \\
\varepsilon \dot{u}=u-u^{3}-\alpha \sinh \left[\frac{v}{\alpha}\right],
\end{gathered}
$$

with $\alpha=2 \omega_{0}^{2} / \mu^{3 / 2}$ and $\varepsilon=2 \omega_{0}^{2} / \mu^{2}$ is a small parameter. Thus we have relaxation oscillations, when slow changes of the variable $u$ are altered by its fast jumps while $v$ performs ramp oscillations (Fig. 5). The "amplitudes" (maximal deviations from zero) are $u_{\max } \simeq 2 / \sqrt{3}, \quad v_{\max }$ $=\alpha \sinh ^{-1}[2 / 3 \sqrt{3} \alpha]$, respectively. Note that the trajectory of the limit cycle largely remains in the domains of "slow" motion (Fig. 5). Approximating the slow motions by $u \approx$ \pm 1 , integration of the first equation of Eq. (19) over the regions of slow motions gives $T \simeq 4 \alpha \sinh ^{-1}[2 / 3 \sqrt{3} \alpha]$ for the oscillation period. Inverting the transformation we get

$$
A_{y}^{\mathrm{st}}=\sinh ^{-1}\left[\frac{\mu^{3 / 2}}{3 \sqrt{3} \omega_{0}^{2}}\right], \quad A_{p}^{\mathrm{st}}=\frac{2 \sqrt{\mu}}{\sqrt{3}}, \quad T^{\mathrm{st}} \simeq \frac{4 A_{y}^{\mathrm{st}}}{\sqrt{\mu}} .
$$

The amplitude of $p$ depends on the "Rayleigh parameter" $\mu$ only. The theoretical estimate of the period of oscillations Eq. (20) in dimensional form gives

$$
T=\frac{4 R_{1} R_{2} C_{2}}{\widetilde{V}} \frac{A_{V}}{R_{d c}},
$$

where $A_{V}$ is the amplitude of the variable $V$ (corresponding to $A_{y}^{\text {st }}$. We note that Eq. (21) does not include the parameters $V_{t}$ and $I_{s}$ which, as we earlier mentioned, cannot be mea- 

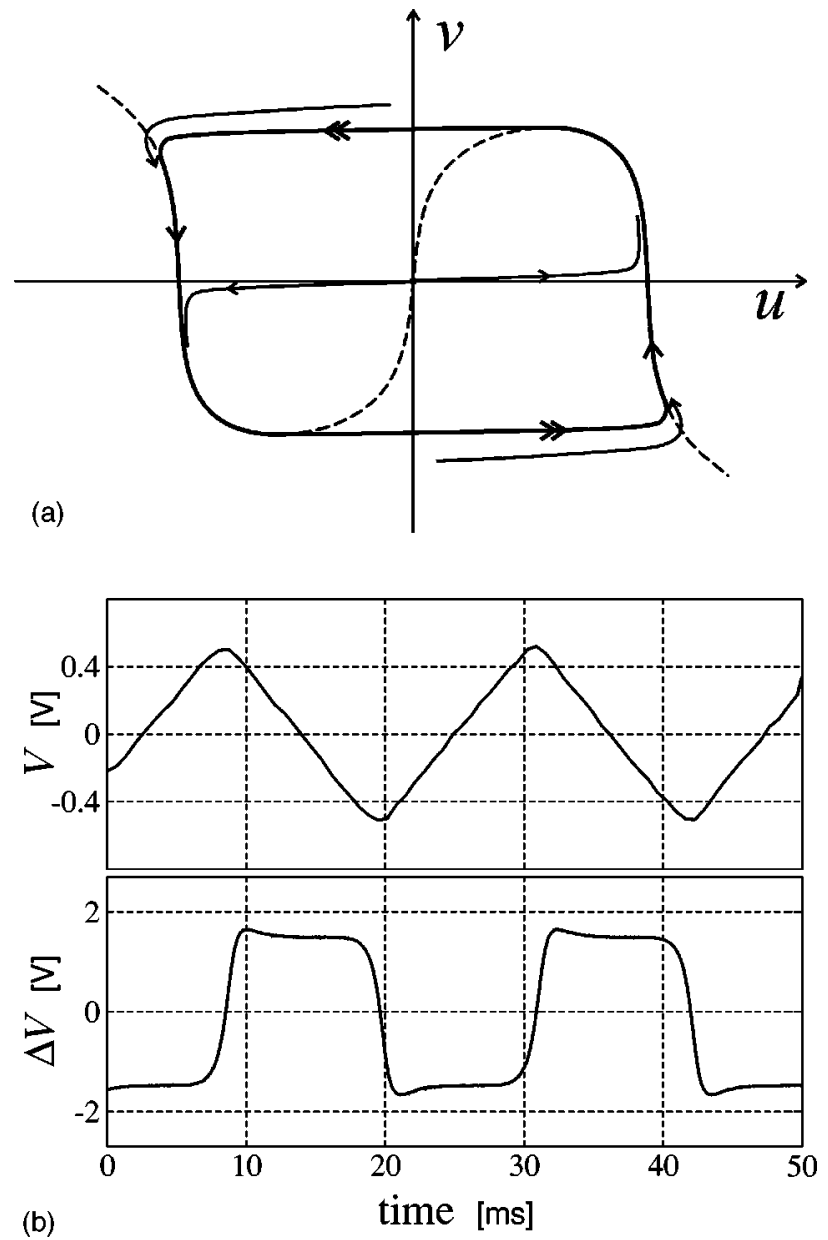

FIG. 5. Dynamics of a single unit: (a) phase plane portrait of a single unit in the case $\mu \gg \omega_{0}$ (single/double arrows on the limit cycle correspond to slow/fast motions; the dashed line corresponds to the curve $\left.v=\alpha \sinh ^{-1}\left[\left(u-u^{3}\right) / \alpha\right]\right)$. (b) Experimental measurement of voltage at the unit $V,(V \propto v)$ and voltage between upper and left-side pins of the double capacitor $\Delta V \propto d V / d t \propto u\left(R_{d c}\right.$ $=681 \Omega, \mu \approx 0.16, \omega_{0} \approx 9 \times 10^{-4}$ ).

sured with precision. A precise measurement of the leading coefficient in Eq. (21) gives $4 R_{1} R_{2} C_{2} / \widetilde{V}=29.32(\mathrm{~s} / \Omega / V)$. According to theory, the period of oscillations Eq. (21) does not depend on $C_{1}$. To check this experimentally in our measurements we used two values: $C_{1}=0.105 \mu F$ and $C_{1}$ $=0.053 \mu F$. For each value of the capacitor $C_{1}$ we change the other parameter of the double capacitor $R_{d c}$, and measure the amplitude and period of oscillations. Figure 6 shows experimental data and the theoretically predicted linear dependence (21). The agreement between theory and experiment is remarkable, both qualitatively and quantitatively, within experimental errors (less than 3\%).

\section{OSCILLATIONS IN THE RING CHAIN}

Earlier, we have numerically obtained different types of oscillations in the system Eq. (3) [33]. It was found that: (i) rotation of a ring as a whole with constant velocity, (ii) soliton-like waves moving clockwise or counterclockwise

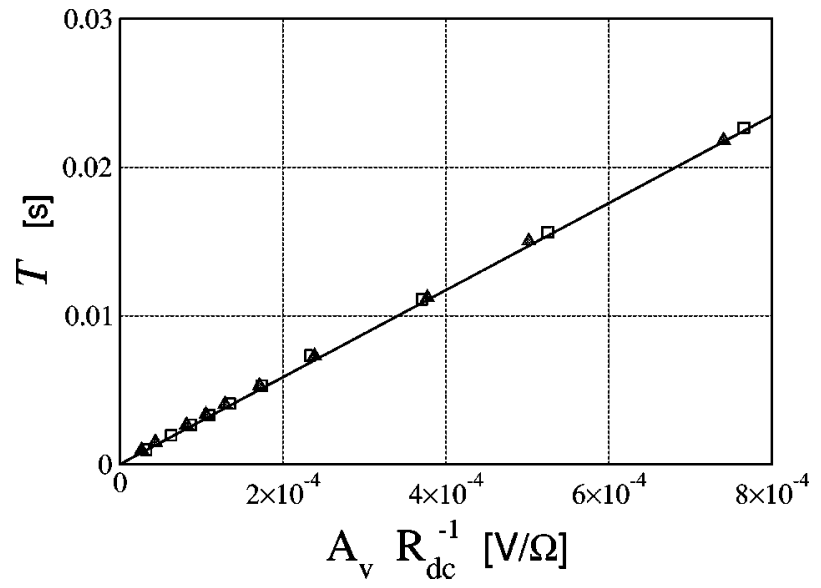

FIG. 6. Oscillation period as a function of the ratio $A_{V} / R_{\mathrm{dc}}$ for two different values of the capacitance $C_{1}$ [Fig. 3(a)]. Squares correspond to $C_{1}=0.105 \mu \mathrm{F}$ and triangles correspond to $C_{1}$ $=0.053 \mu F$. The straight line is the theoretical estimate of the period Eq. (21).

"upstream" (opposite to the direction of average mass rotation), and (iii) short-length scale oscillations. On the other hand, for a system similar to Eq. (1) with a special form of the function $G$ and for high values of $B$, numerical computations show that the system possesses $(N+1)$ attracting sets that differ by the total momentum $P=1 / N \Sigma_{n=1}^{N} P_{n}$ [48]. Here we shall proceed with a systematic analysis of the possible modes of oscillations in the system Eq. (3). In the previous section we have investigated the dynamics of a single, isolated unit, and found that for positive $\mu$ it always produces oscillations. Let us now study the dynamics of the ring chain. For a closed ring we have the following boundary conditions:

$$
y_{n+N}(t)=y_{n}(t)
$$

where $N$ is the total number of units.

For any positive $\mu$ the system Eq. (3) admits three nonvibrating spatially homogeneous solutions:

$$
\begin{aligned}
& \text { (i) } \quad x_{n}=0, p_{n}=0, \\
& \text { (ii) } x_{n}=0, p_{n}=\sqrt{\mu}, \\
& \text { (iii) } x_{n}=0, \quad p_{n}=-\sqrt{\mu} .
\end{aligned}
$$

The first solution is unstable and the other two are stable [33]. These stable solutions correspond to clockwise and counterclockwise rotations of the ring as a whole with corresponding velocity $\pm \sqrt{\mu}$. In the electrical circuit they can be observed by adjusting $V_{\text {ext }}= \pm \widetilde{V}$ (Fig. 2) so that $d V_{n} / d t$ $=0$.

\section{A. Space period-2 oscillations: Experiments with a ring of two units and the role of the resistor $R$}

Let us first consider the simplest oscillatory solutions occurring in the ring Eq. (3) with an even number of units. The 


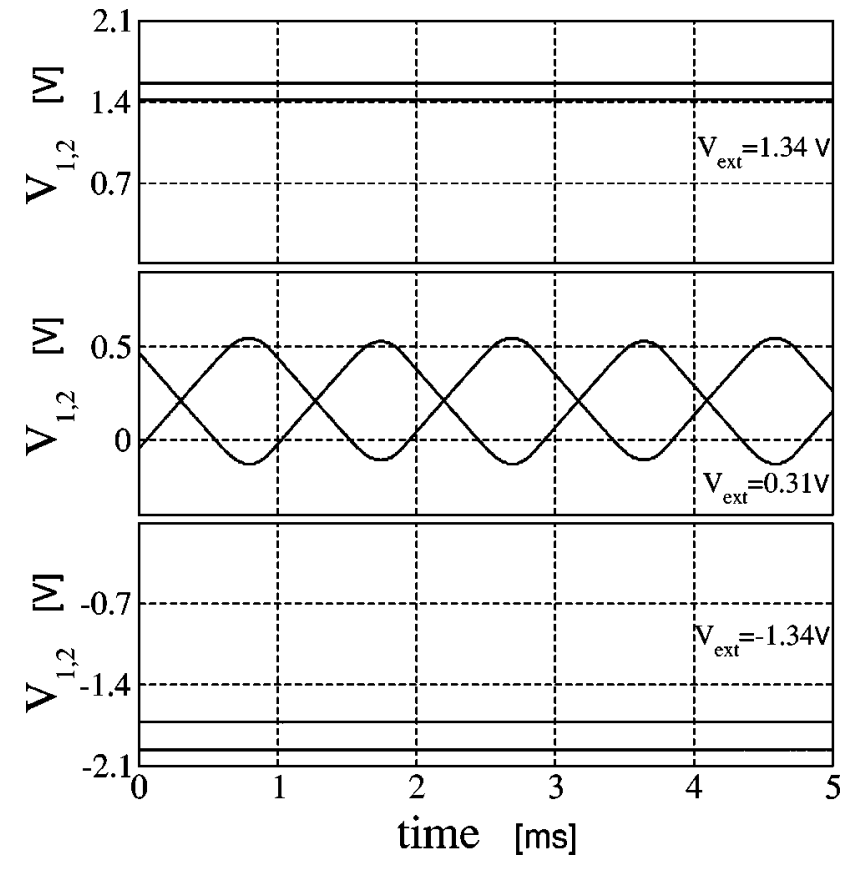

FIG. 7. Ring with two units. Experimental voltages at the units for different values of $V_{\text {ext }}$, corresponding to two nonoscillatory modes (top and bottom) and the optical mode (center region) $\left(R_{d c}\right.$ $=13681 \Omega, \mu \approx 3.75 \times 10^{-4}, \omega_{0} \approx 10^{-5}$ ).

shortest "ring" is a chain of two units coupled by two oppositely connected diodes, such that $y_{0}=y_{2}$ and $y_{3}=y_{1}$.

Let us now assume that we have the space period- 2 solution

$$
y_{n}(t)=y_{n+2}(t)
$$

Changing variables $s=p_{n-1}+p_{n}$ and $r=p_{n-1}-p_{n}$, from Eqs. (3) and (23) we have

$$
\begin{gathered}
\dot{x}=r, \\
\dot{r}=-4 \omega_{0}^{2} \sinh x+r\left[\mu-\frac{1}{4}\left(r^{2}+3 s^{2}\right)\right], \\
\dot{s}=s\left[\mu-\frac{1}{4}\left(3 r^{2}+s^{2}\right)\right] .
\end{gathered}
$$

For the shortest ring $(N=2)$, Eqs. (24) give a full description of all possible motions. For rings with $N>2$ units, Eqs. (24) are determined in the three-dimensional manifold of the corresponding $2 N-1$ dimensional phase space.

For $\mu \leqslant 0$ the rest state is the only attractor. For positive $\mu$ the system Eq. (24) has (see Appendix A) three attractors: (i) two steady states $\left(x_{n}=0, p_{n}= \pm \sqrt{\mu}\right)$ corresponding to constant rotations with velocities $c= \pm \sqrt{\mu}$, and (ii) a limit cycle in the plane $s=0$. The limit cycle corresponds to antiphase oscillations in neighboring units (the so-called "optical mode") with $c=0$. Figure 7 shows oscilloscope traces for these three modes. They have been obtained for different values of external voltages. At the beginning, we fixed $V_{\text {ext }}$ and one of the three modes was formed in the system. The other two modes were obtained by changing $V_{\text {ext }}$. For specific external voltages the system jumped from one mode to the other.

Let us now discuss the stabilizing role of the resistor $R$ (Fig. 2). We have mentioned above that the external voltage $V_{\text {ext }}$, which helps in eliminating the effect of a "constant rotation," has to be differently tuned for each mode. In principle we have to choose the exact values of the external voltage $V_{\text {ext }}=\{0,-\widetilde{V}, \widetilde{V}\}$ to avoid saturation in the circuit. However, such fine tuning is impossible in practical terms. Any small differences in the electronic components, unavoidable spontaneous fluctuations, or noise will lead to deviation from ideality, and consequently, to a relatively slow voltage drift that finally saturates the circuit. The resistor $R$ does not permit these slow changes by expanding the range of values of $V_{\text {ext }}$ valid for the correct circuit operation from points (exact values) into intervals. To show this let us analyze the influence of the resistor on the dynamics of the ring. Besides, here we take into account that the dissipative function $G$ depends on the external voltage $V_{\text {ext }}$. From Eq. (16) we have

$$
\begin{aligned}
& \ddot{y}_{1}=\omega_{0}^{2}\left(e^{y_{2}-y_{1}}-e^{y_{1}-y_{2}}\right)+f\left(\dot{y}_{1}-p_{\text {ext }}\right)-\omega_{R}^{2} y_{1}, \\
& \ddot{y}_{2}=\omega_{0}^{2}\left(e^{y_{1}-y_{2}}-e^{y_{2}-y_{1}}\right)+f\left(\dot{y}_{2}-p_{\text {ext }}\right)-\omega_{R}^{2} y_{2},
\end{aligned}
$$

where $f(x)=\left(\mu-x^{2}\right) x$ is the nonlinear function and $p_{\text {ext }}$ $=\omega_{v}^{2} R_{\mathrm{dc}} C_{1} V_{\text {ext }}[$ see Eq. (12)]. Let us now assume that the value $p_{\text {ext }}$ (or, in practical terms, $V_{\text {ext }}$ ) is tuned such that for a certain mode the constant voltage drift disappears. Then

$$
\left\langle p_{1,2}(t)\right\rangle_{t}=\frac{1}{T} \int_{0}^{T} \dot{y}_{1,2}(t) d t=y_{1,2}(T)-y_{1,2}(0) \equiv 0,
$$

where $T$ denotes the period of the oscillations. Averaging Eq. (25) we have for the mean value of the variables $y_{1}$ and $y_{2}$

$$
\bar{y}=\frac{\left\langle y_{1}+y_{2}\right\rangle_{t}}{2}=\frac{\left\langle f\left(\dot{y}_{1}-p_{\mathrm{ext}}\right)+f\left(\dot{y}_{2}-p_{\mathrm{ext}}\right)\right\rangle_{t}}{2 \omega_{R}^{2}} .
$$

For nonoscillatory modes $\left(\dot{y}_{1,2}=0\right)$, from Eq. (26) we have a solution of Eq. (25)

$$
y_{1}=y_{2}=\bar{y}_{\mathrm{hom}}=-\frac{f\left(p_{\mathrm{ext}}\right)}{\omega_{R}^{2}} .
$$

To have the electrical circuit working, the voltage ( $V \propto y$ ) has to be inside the operating regime, $\left|y_{1,2}\right|<y_{\text {sat }}$. Hence, for $R$ $\rightarrow \infty\left(\omega_{R} \rightarrow 0\right)$ we have to demand $f\left(p_{\text {ext }}\right) \rightarrow 0$, which corresponds to perfect tuning of $V_{\text {ext }}$. For finite $R$, the circuit will not saturate for the external voltage inside the intervals

$$
\left|p_{\text {ext }}\right| \lesssim \frac{\omega_{R}^{2} y_{\text {sat }}}{\mu}, \quad\left|p_{\text {ext }} \pm \sqrt{\mu}\right| \lesssim \frac{\omega_{R}^{2} y_{\text {sat }}}{2 \mu}
$$

that can be experimentally satisfied.

Linearizing Eq. (25) near solutions Eq. (27), we obtain the following eigenvalues: 


$$
\begin{gathered}
\lambda_{1,2}=\frac{1}{2}\left[f^{\prime}\left(p_{\text {ext }}\right) \pm \sqrt{f^{\prime 2}\left(p_{\text {ext }}\right)-4 \omega_{R}^{2}}\right], \\
\lambda_{3,4}=\frac{1}{2}\left[f^{\prime}\left(p_{\text {ext }}\right) \pm \sqrt{f^{\prime 2}\left(p_{\text {ext }}\right)-4 \omega_{R}^{2}-16 \omega_{0}^{2}}\right],
\end{gathered}
$$

and hence they are stable for $f^{\prime}\left(p_{\text {ext }}\right)<0$. There exist two branches of the function $f\left(p_{\text {ext }}\right)$ (Fig. 4) with negative derivative: $p_{\text {ext }}<-\sqrt{\mu / 3}$ and $p_{\text {ext }}>\sqrt{\mu / 3}$. Positioning $V_{\text {ext }}$ inside these regions and intervals Eq. (28) we can observe two nonoscillatory modes.

For the optical mode we get from Eq. (26)

$$
\bar{y}_{\mathrm{opt}}=\frac{-f\left(p_{\text {ext }}\right)+3 p_{\text {ext }}\left\langle\dot{y}_{1,2}^{2}\right\rangle}{\omega_{R}^{2}} .
$$

Let us estimate the value $\left\langle\dot{y}_{1,2}^{2}\right\rangle$. For $y_{1,2}(t)$ we can write $y_{1}(t)=\bar{y}_{\mathrm{opt}}+v(t)$ and $y_{2}(t)=\bar{y}_{\mathrm{opt}}-v(t)$ with $\langle v\rangle \equiv 0$. Then from Eq. (25) we have

$$
\begin{gathered}
\dot{v}=u+p_{\text {ext }}, \\
\dot{u}=-2 \omega_{0}^{2} \sinh [2 v]+f(u)-\omega_{R}^{2}\left(\bar{y}_{\mathrm{opt}}+v\right) .
\end{gathered}
$$

Now we can write

$$
\left\langle\dot{y}_{1,2}^{2}\right\rangle_{t}=\left\langle\dot{v}^{2}\right\rangle_{t}=\frac{1}{T} \int_{0}^{T}\left(u(t)+p_{\text {ext }}\right)^{2} d t=\frac{1}{T} \oint u(v) \mathrm{d} v,
$$

where $T$ is the period of the oscillations. Assuming that $\omega_{0}$ $\ll \mu$ we can calculate $T$ as in Sec. III and, consequently, the integral. We obtain $\left\langle\dot{y}_{1,2}^{2}\right\rangle_{t} \simeq \mu-p_{\text {ext }}^{2}$. Thus substituting this result into Eq. (29) we get an estimate for the mean value of the voltage

$$
\bar{y}_{\mathrm{opt}}=\frac{2 f\left(p_{\mathrm{ext}}\right)}{\omega_{R}^{2}} .
$$

Again, to have finite mean voltage value (inside circuit operation region) for the imperfectly tuned $V_{\text {ext }}$ we need to require that $\omega_{R} \neq 0$. Finally, the optical mode exists for $\left|p_{\text {ext }}\right|<\sqrt{\mu}$. This domain of $p_{\text {ext }}$ intersects with the domain of stability of the nonvibrating modes, $\left|p_{\text {ext }}\right|>\sqrt{\mu / 3}$.

From Eqs. (27) and (32) it follows that both nonoscillatory and optical modes have linear dependencies of the mean voltage $(\bar{V} \propto \bar{y})$ on the current via the nonlinear resistor $\left(I_{\mathrm{nr}}\right.$ $\propto f$ ). Experimental measurements of the mean voltage value $\bar{V}$ for different solutions as a function of the current via the nonlinear resistor $I_{\mathrm{nr}}$ are shown in Fig. 8. The data have been obtained with less than $3 \%$ relative error. In agreement with theory we have linear dependency both for nonoscillatory solutions and the optical mode. The two lines marked by triangles correspond to two non-oscillatory solutions. The small mismatch between them is due to no strict identity of the units and a small asymmetry of the nonlinear function $I_{\mathrm{nr}}$. The slope of these straight lines is $-104 \mathrm{k} \Omega$. Theory

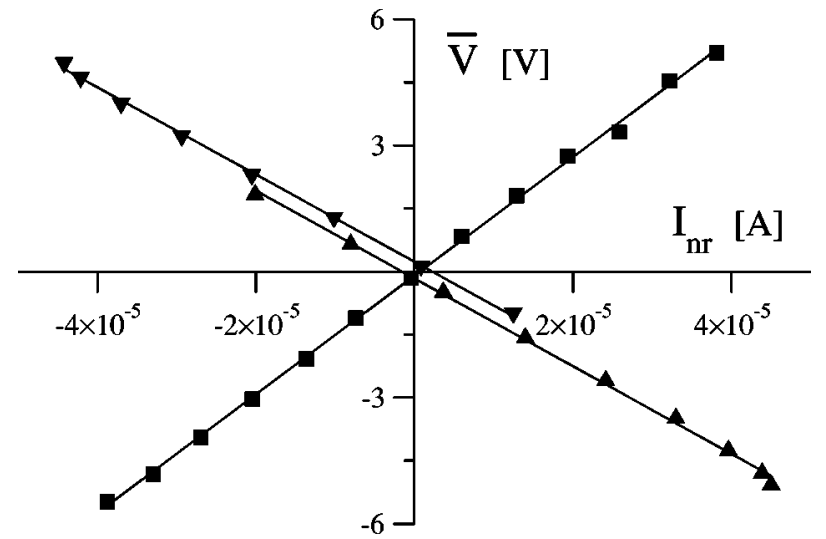

FIG. 8. Average voltage on the units $\bar{V}$ vs current through the nonlinear resistor $I_{\mathrm{nr}}$ for the optical mode (squares) and for the homogeneous solutions (up/down triangles correspond to "left"/ "right" rotations).

gives the value $R=\bar{V} / I_{\mathrm{nr}}=-120 \mathrm{k} \Omega$. The $13 \%$ difference is explained by the nonzero current via the double capacitor even if the voltage applied is constant. Our measurements show that the leakage current $I_{\mathrm{dc}} \approx 8 \mu \mathrm{A}$ in this case, which corresponds to a decreasing effective resistance from $120 \mathrm{k} \Omega$ to $104 \mathrm{k} \Omega$. For the optical mode, theory predicts a slope of $240 \mathrm{k} \Omega$ [Eq. (32)] but experimentally we got $141 \mathrm{k} \Omega$. This discrepancy is again due to an additional current via the double capacitors and the approximations used to compute the period and integral in Eq. (31).

To conclude this section we should mention that the additional term proportional to $y_{n}$ in the Toda-Rayleigh model due to the resistor $R$ leads to the appearance of a new attractor. This attractor does not exist in the approximation $R$ $\rightarrow \infty$. Substituting the ansatzs $y_{1}=y_{2}=y(t)$ into Eq. (25) we get for $y(t)$

$$
\ddot{y}+\omega_{R}^{2} y=f\left(\dot{y}-p_{\text {ext }}\right) .
$$

Equation (33) has a stable limit cycle. Its large amplitude precludes its experimental observation as the circuit saturates and we hence disregard it from our present study.

\section{B. $N-1$ oscillatory modes (bifurcation at $\mu=0$ )}

Earlier we have investigated some special cases. We have shown that in a "ring" of two units for $\mu>0$ only one oscillatory (optical) mode exists. Now let us study the bifurcation at $\mu=0$ for a ring consisting of an arbitrary number of units. We shall consider $\mu$ as the significant bifurcation parameter.

Let us consider the function

$$
H(\mathbf{y}, \mathbf{p})=\sum_{n=1}^{N}\left[\frac{p_{n}^{2}}{2}+\omega_{0}^{2}\left(e^{x_{n}}-1-x_{n}\right)\right],
$$

which is the Hamiltonian for the conservative case $(G=0)$. The time derivative of $H(\mathbf{y}, \mathbf{p})$ along the solution curves of Eq. (3) is 


$$
\frac{d H}{d t}=\sum_{n=1}^{N}\left(\mu-p_{n}^{2}\right) p_{n}^{2}
$$

For $\mu \leqslant 0$, this derivative Eq. (35) is less or equal to zero $\left(d H / d t=0\right.$ only if all $\left.p_{n}=0\right)$. Hence in this case the function $H(\mathbf{y}, \mathbf{p})$ can be considered as a Lyapunov function. Consequently, the motionless state $\left\{p_{n}=0, y_{n+1}-y_{n}=0\right\}$ is globally asymptotically stable.

For $\mu>0$ the derivative Eq. (35) is positive definite in the domain $\left|p_{n}\right|<\sqrt{\mu}$. Hence all trajectories of Eq. (3) cross the level surfaces

$$
p_{n}^{2}+2 \omega_{0}^{2}\left(e^{x_{n}}-1-x_{n}\right)=h, \quad h<\mu, \quad n=1,2, \ldots, N
$$

outwards. Thus the rest state $\left\{p_{n}=0, x_{n} \equiv y_{n}-y_{n-1}=0\right\}$ is unstable and, moreover, there is no attractor inside the domain bounded by the surface Eq. (36) with $h=\mu$.

Let us now investigate the bifurcation at $\mu=0$. First, we investigate the linear case. Introducing perturbations around the steady state $\left(p_{n}=x_{n}=0\right) y_{n}=\xi_{n}, p_{n}=\eta_{n}$ and using their Fourier representation

$$
\begin{gathered}
\xi_{n}=\sum_{m=1}^{N} \widetilde{\xi}_{m} e^{i M n}, \quad \eta_{n}=\sum_{m=1}^{N} \tilde{\eta}_{m} e^{i M n}, \\
M=\frac{2 \pi m}{N}, \quad m=1,2, \ldots, N
\end{gathered}
$$

we get from Eq. (3) $N$ uncoupled linear second-order equations for the space Fourier amplitudes

$$
\begin{gathered}
\dot{\vec{\xi}}_{m}=\tilde{\eta}_{m}, \\
\dot{\tilde{\eta}}_{m}=-2 \omega_{0}^{2}(1-\cos M) \widetilde{\xi}_{m}+\mu \tilde{\eta}_{m} .
\end{gathered}
$$

Thus the stability analysis in the $2 N$-dimensional system drastically simplifies. The eigenvalues corresponding to Eq. (38) are given by

$$
\lambda_{1,2}^{m}=\frac{\mu \mp \sqrt{\mu^{2}-8 \omega_{0}^{2}(1-\cos M)}}{2} .
$$

One eigenvalue $\lambda_{1}^{N}$ (for $m=N$ ) vanishes due to the translation symmetry of the system. Another is real $\left(\lambda_{2}^{N}=\mu\right)$ and changes sign as $\mu$ also changes sign. The other $2(N-1)$ roots have negative real part for $\mu<0$ and, simultaneously, cross the imaginary axis at $\mu=0$, and for $\mu>0$ we have an unstable steady state as we have already seen. At $\mu=0$ we have $(N-1)$ pairs of imaginary roots with frequencies of linear oscillations

$$
\omega_{m}=2 \omega_{0} \sin \frac{\pi m}{N}, \quad m=1,2, \ldots, N-1
$$

that for $m=N$ vanishes. This corresponds to two nonoscillating solutions Eq. (22) ("clockwise" or "counterclockwise" homogeneous rotations) of the system Eq. (3). Thus we have a kind of symmetric Hopf bifurcation.

Using the results of the linear analysis let us now consider the case $|\mu| \ll \min \left\{\omega_{m}, \omega_{m}^{2}\right\}$. Further we shall require that $|\mu| \ll \min \left\{\omega_{0} \sin \pi / N, \omega_{0}^{2} \sin ^{2} \pi / N\right\}$. This condition is rather difficult to be fulfilled by very long ring chains. Hence we assume that the ring consists of a suitably low number of units.

For positive $\mu$ we expect the appearance of low amplitude oscillations with a frequency near the linear value. Accordingly, we set

$$
\begin{gathered}
y_{n}=\frac{\sqrt{\mu} a}{\omega_{m}} \cos \left(\omega_{m} t+M n+\varphi\right), \\
p_{n}=-\sqrt{\mu} a \sin \left(\omega_{m} t+M n+\varphi\right),
\end{gathered}
$$

where $a$ and $\varphi$ are the amplitude and the phase of oscillations. They are slow functions of time. In this notation $m$ can be treated as a wave number. Then, the wavelength is $N / m$.

Substituting the variable transformation Eq. (40) into the system Eq. (3), changing time, $t_{\text {new }}=\omega_{m} t_{\text {old }}$, and expanding the exponentials we obtain

$$
\begin{aligned}
& \dot{a}=\left[\frac{\sqrt{\mu} a^{2}}{2 \omega_{0}} F(a, \theta)+\frac{\mu a}{\omega_{m}}\left(\sin \theta-a^{2} \sin ^{3} \theta\right)\right] \sin \theta, \\
& \dot{\varphi}=\left[\frac{\sqrt{\mu} a}{2 \omega_{0}} F(a, \theta)+\frac{\mu}{\omega_{m}}\left(\sin \theta-a^{2} \sin ^{3} \theta\right)\right] \cos \theta,
\end{aligned}
$$

with

$$
\begin{gathered}
\theta=\omega_{m} t+M n+\varphi \\
F=\cos \frac{M}{2} \sin 2 \theta+\frac{\sqrt{\mu} a}{12 \omega_{0}}(3 \cos \theta-\cos 3 \theta(1+2 \cos M)) .
\end{gathered}
$$

Now we can apply the averaging method to the system Eq. (41) and obtain

$$
\begin{gathered}
\dot{a}=\frac{\mu}{2 \omega_{m}} a\left(1-\frac{3}{4} a^{2}\right) \\
\dot{\varphi}=\frac{\mu}{16 \omega_{0}^{2}} a^{2} .
\end{gathered}
$$

The system Eq. (42) has an unstable steady state at the origin and a stable limit cycle with amplitude $a=2 / \sqrt{3}$.

Thus in the ring we have $(N-1)$ different stable oscillatory modes with the following stationary amplitudes and frequencies:

$$
\begin{gathered}
A_{y}^{m}=\frac{\sqrt{\mu}}{\sqrt{3} \omega_{0} \sin \frac{\pi m}{N}}, \quad A_{p}^{m}=\frac{2 \sqrt{\mu}}{\sqrt{3}}, \\
\omega_{\mathrm{st}}^{m}=2 \omega_{0} \sin \frac{\pi m}{N}\left(1+\frac{\mu}{12 \omega_{0}^{2}}\right) .
\end{gathered}
$$




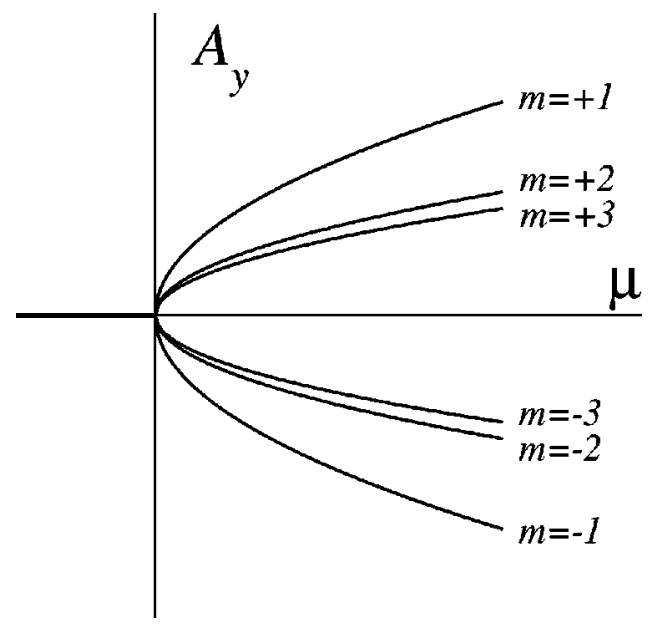

FIG. 9. Schematic diagram of mode amplitudes Eq. (43) as a function of bifurcation parameter for different mode number $m(N=6)$.

Besides, there exist two trivial states with $p_{n}= \pm \sqrt{\mu}$. Thus the system Eq. (3) for $0<\mu \ll \omega_{0} \sin \pi / N$ has $(N+1)$ different stable states: $(N-1)$ oscillatory modes and two nonoscillatory, rotatory modes. For the momenta $p_{n}$ the amplitude of oscillations is the same for all modes. However for the voltage variable $y$ the amplitude depends on the wave number. The modes $m=(N-1),(N-2), \ldots$ have the same amplitude as the modes with $m=1,2, \ldots$ They correspond to waves with the same space scales like waves with $m$ $=1,2, \ldots$, but propagating in opposite directions. We can denote them as modes having negative mode number $m=$ $-1,-2, \ldots$ For even $N$ there is a "special" (optical) mode with $m= \pm N / 2$ (both signs define the same wave form). In such modes, neighboring units oscillate in anti-phase as already studied in Sec. IV A. The two longest (propagating in opposite directions) modes with $m= \pm 1$ have the largest amplitude. The amplitude decreases with the increase of mode number. Figure 9 illustrates the bifurcation occurring at $\mu$ $=0$. For positive $\mu$ we have $N-1=5$ different waves $(m$ $= \pm 3$ corresponds to one and the same optical mode). Negative amplitude values correspond to a phase shift of $\pi$ and to waves propagating in the opposite direction. Depending on initial conditions, after a transient time one of the seven (5 oscillatory +2 nonoscillatory) modes appears in the ring. Equations (43) are valid for small enough $\mu$. With increasing $\mu$, oscillations become stiffer and the amplitude does not follow the $\sqrt{\mu}$ law.

To visualize the experimental results several variables such as currents through diodes $I_{n}$, voltages at the units $V_{n}$, or their time derivatives $d V_{n} / d t$ can be used. For example, in [36] currents through diodes were measured by placing a small resistor $(1 \Omega)$ in series with each diode. Figure 10 shows our measurements of the voltage on the resistor, i.e., current, by a 12-bit digital oscilloscope NICOLET-420. However, due to the exponential voltage-current characteristics of diodes, these variables show practically the same behavior for all modes (Fig. 10). Even, the optical mode ( $m$ $=3$ ), whose voltage trace is rather close to harmonic oscillations (Fig. 7), looks like the longest mode with $m=1$ that

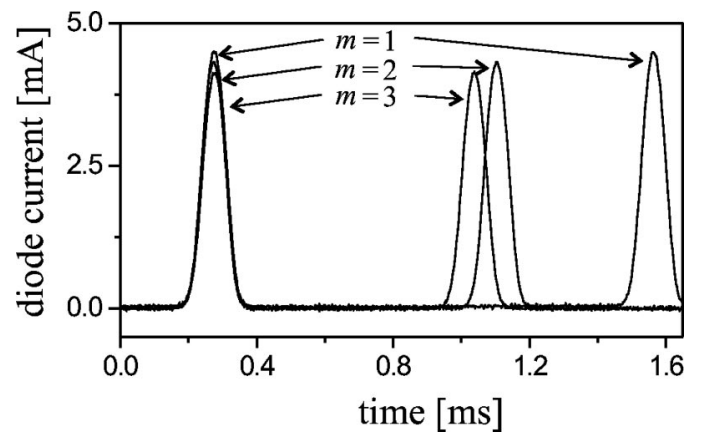

FIG. 10. Oscilloscope traces of current through a diode for different modes in the ring of six units. For each mode the first peak has been positioned at the same instant of time $\left(V_{\text {ext }}=0.45 \mathrm{~V}\right.$, $R_{d c}=13681 \Omega, \mu \approx 3.75 \times 10^{-4}, \omega_{0} \approx 10^{-5}$ ).

represents a soliton solution in the limit of a long enough chain. Thus we have chosen the voltage and its time derivative to illustrate the dynamics of the circuit (in the original Toda lattice the soliton shape solution is observed for the time derivative of displacement variables or for their nearestneighbor differences).

Figure 11 shows experimental traces for three different modes taken for the same external voltage. The $m=1$ wave has the largest amplitude while waves with $m=2,3$ have about the same amplitude as theoretically predicted (Fig. 9). Besides, the shape and the mean values of voltages are clearly different for all modes. Figure 12 shows voltage differences, $V_{n}-V_{b}$, taken from all units (Fig. 2). These voltage differences are proportional to the time derivatives of voltages $d V_{n} / d t$ [see Eq. (11)]. Due to the designed leftright symmetry of the system we have two similar waves propagating clockwise and counterclockwise. The clockwise [Fig. 11(a)]/counterclockwise [Fig. 11(b)] waves propagate over some negative/positive base levels and have positive/

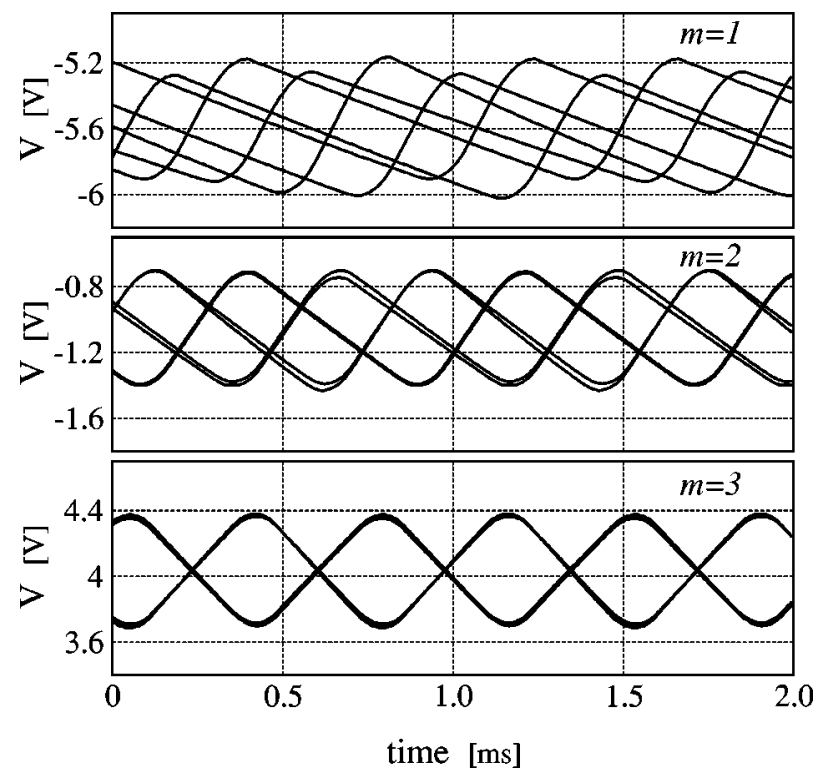

FIG. 11. Oscilloscope traces of the voltages taken from all six units of the ring for three different modes. All parameter values are the same as for Fig. 10. 


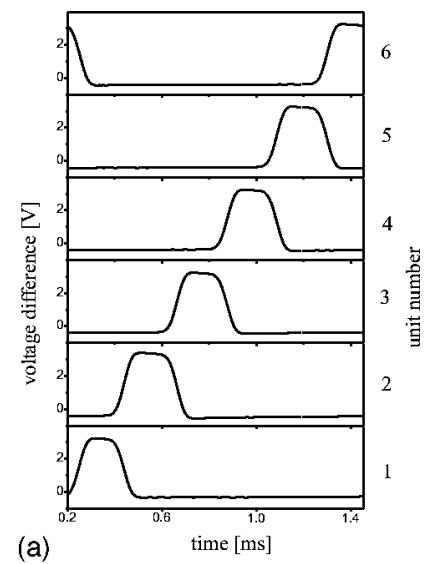

(a)

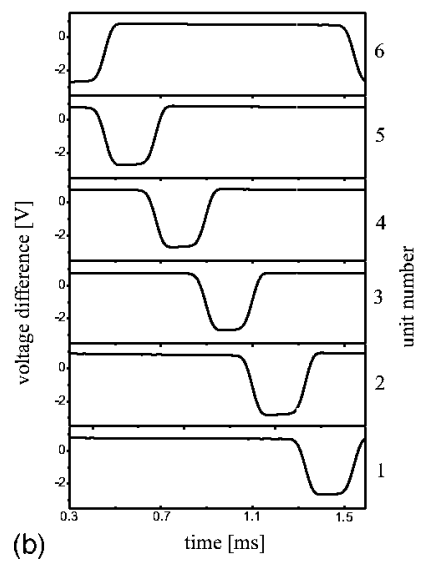

FIG. 12. Oscilloscope traces showing propagation of the two longest waves: (a) wave running clockwise, $m=1 \quad\left(V_{\mathrm{ext}}\right.$ $=0.45 \mathrm{~V})$ and (b) wave running counterclockwise, $m=-1 \quad\left(V_{\text {ext }}\right.$ $=0.45 \mathrm{~V})$. The $Y$ axes provide voltage differences between voltage on the unit and left side pin of the double capacitor (Fig. 2) that is proportional to the time derivative of $V_{n}(t) \mathrm{Eq}$. (11). The total time interval corresponds to the oscillation period. All parameter values (excluding $V_{\text {ext }}$ ) are the same as on Figs. 10 and 11.

negative humps as theory predicts.

Due to the multistability in the system, we can observe different modes for the same parameter set. Such a situation can lead to competition of waves. We used $V_{\text {ext }}$ as a parameter to study this phenomenon. We started from the nonoscillatory mode and a high, negative value of $V_{\text {ext }}(-1.8 \mathrm{~V})$. Then we slowly increase $V_{\text {ext }}$ to high positive values and follow the mode dynamics. Figure 13 shows the results of measurement of mode stability and the jumps that occur between modes. Several modes can overlap for the same value of the external voltage. Thus as $V_{\text {ext }}$ increases beyond a critical point the initial mode becomes unstable. For that value of $V_{\text {ext }}$ we have two other modes that are stable. Hence one of them can be the winner in the competition. For example, for $V_{\text {ext }} \geq-0.85 V$ the $m=-0$ (nonoscillatory mode with "left" rotation) becomes unstable but the two others with $m=-1,-2$ are stable. Then either the mode with $m=-1$ or the $m=-2$ mode can win and be established in the ring. In our experiments we observed both possibilities in different runs. Further increasing $V_{\text {ext }}$ leads to new jumps between modes as shown in Fig. 13. Analogous jumps are observed

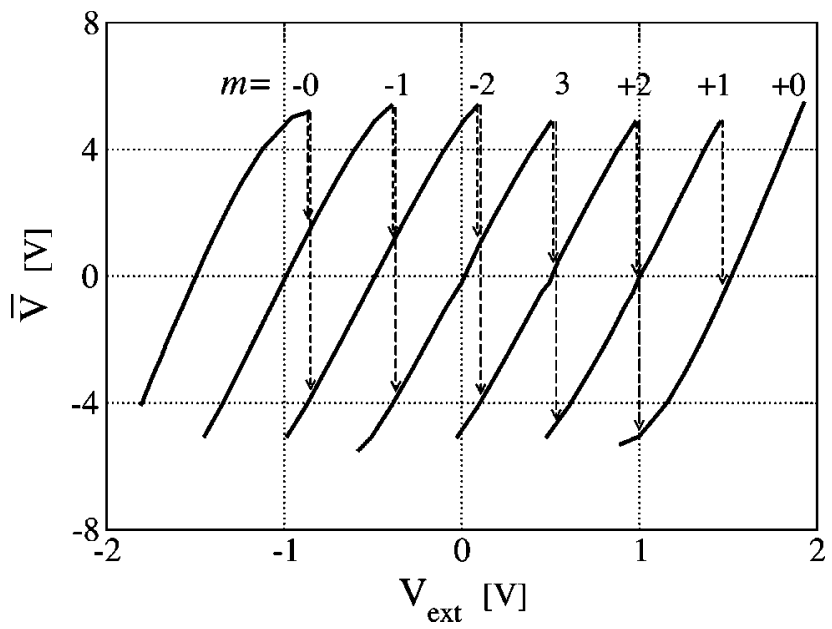

FIG. 13. Average voltage on units for different modes and direction of jumps between them vs the external voltage $V_{\text {ext }}$ in the ring of six units. Each curve corresponds to a certain mode with mode number marked $m$ on its corresponding top $(R=120 \mathrm{k} \Omega)$.

while decreasing the external voltage from $1.8 \mathrm{~V}$ to $-1.8 \mathrm{~V}$ (not shown in Fig. 13).

In practical terms, in contrast to what we have seen in Fig. 13 , the absence of uncertainty in mode formation and jumps between them is usually preferable. We found experimentally that by decreasing the value of the resistor $R$ to its half value (from 120 to $60 \mathrm{k} \Omega$ ) we have a situation without uncertainty in jumps between modes. Namely, the jumps occur between two nearest modes only and there are no jumps to the next nearest neighbors. Figure 14 shows the sequence of all jumps between modes. Clearly, there is an hysteresis-like behavior between any two nearest modes. Changing the value of the external voltage we can get the desired mode.

\section{CONCLUSION}

We have studied the formation and propagation of waves, and switching between different wave modes, in a dissipative

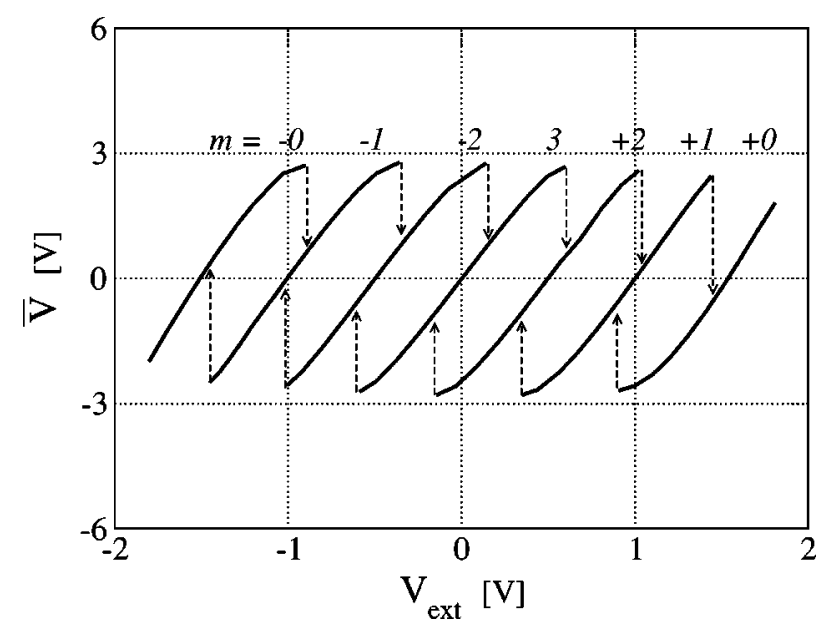

FIG. 14. Jumping sequence between modes for low values of the resistor $R=60 \mathrm{k} \Omega$ (Fig. 2). The remaining parameter values are the same as for Fig. 13. 
Toda-Rayleigh system. A rather general Rayleigh type of energy pumping-dissipation balance was used. We have shown up to what extent new phenomena appear that the original Toda lattice cannot exhibit. A finite number of stable wave modes exists and grows with the increase in the number of units as $N-1$. For a long enough ring the longest modes with $m= \pm 1, \pm 2, \ldots$ transform into soliton-like waves having $|m|$ crests. All crests have the same "polarization" and propagate in the same direction. Initial conditions permit that a certain wave finally forms in the ring. However, its actual shape is defined by the energy balance and does not depend on the initial conditions. We have also studied the bifurcation leading to wave multistability. For a prime number of units (e.g., $N=5$ ) we have found that all $(N-1)$ modes have the same oscillatory regime in each unit. The modes differ by a phase shift between units only. For example, for $N=5, m$ $= \pm 2$, phase growth occurs along the ring in a time sequence forming an imaginary five-ray star $(1-3-5-2-4-1)$.

Theory has been complemented with experiments using an electronic implementation of the model with a novel method to stabilize a stationary rotating wave without the need of resetting the setup after each period. For rings with five and six units we experimentally observed all wave modes predicted by the theory. We have also studied jumps between different modes while the common external voltage changes. Besides, we have experimentally found the value of the resistor $R(60 \mathrm{k} \Omega)$ when our circuit allows us to choose between different modes by playing with only one external voltage, whatever the initial conditions in the units may be. This property of the circuit can be used in applications for formation and control of various spatio-temporal patterns without changing the geometry or the internal structure of the system.

\section{ACKNOWLEDGMENTS}

The authors acknowledge fruitful discussions with Dr. L. P. Shilnikov. V.A.M. benefitted from financial support from the Spanish Ministry of Science and Technology and W.E. from the Humboldt-Mutis Fund. This work was supported by the Spanish Ministry of Science and Technology under Grant Nos. PB 96-599 and PB 97-0574-C04-01.

\section{APPENDIX A:}

Let us show that the system Eq. (24) has only three attractors for positive $\mu$.

The plane $s=0$ is the integral surface of Eq. (24). This plane divides the phase space into two symmetrical parts. On this plane we have

$$
\dot{x}=r, \quad \dot{r}=-4 \omega_{0}^{2} \sinh x+\mu r-\frac{1}{4} r^{3},
$$

which correspond to the equation for a single unit Eq. (17). Accordingly, we have a stable (at least to perturbations inside the plane) limit cycle.

Let us investigate the dynamics of Eq. (24) when $\mu$ $\ll \min \left\{\omega_{0}, \omega_{0}^{2}\right\}$. We can introduce new variables $x$ $=\left(\sqrt{\mu} / 2 \omega_{0}\right) w, r=\sqrt{\mu} u, s=\sqrt{\mu} z, t_{\text {new }}=2 \omega_{0} t_{\text {old }}$, and expand $\sinh \left[\left(\sqrt{\mu} / 2 \omega_{0}\right) w\right]$. Then from Eq. (24) we get

$$
\begin{gathered}
\dot{w}=u, \\
\dot{u}=-w+\varepsilon\left[u\left(1-\frac{1}{4}\left(u^{2}+3 z^{2}\right)\right)-\frac{1}{12 \omega_{0}} w^{3}\right], \\
\dot{z}=\varepsilon z\left(1-\frac{1}{4}\left(3 u^{2}+z^{2}\right)\right),
\end{gathered}
$$

with $\varepsilon=\mu / 2 \omega_{0}$. Now introducing amplitude-phase $(a, \varphi)$ variables, $w=2 a \cos (t+\varphi), u=-2 a \sin (t+\varphi)$, and averaging over the period of oscillations we obtain

$$
\begin{gathered}
\dot{a}=\frac{\varepsilon}{2} a\left[1-\frac{3}{4}\left(a^{2}+z^{2}\right)\right], \\
\dot{z}=\varepsilon\left[1-\frac{1}{4}\left(6 a^{2}+z^{2}\right)\right], \\
\dot{\varphi}=\frac{\varepsilon}{8 \omega_{0}} a^{2} .
\end{gathered}
$$

The first two equations, Eq. (A3), are independent of the third one, Eq. (A4), and we can consider them separately.

On the plane $(a, z)$ the vector field is invariant to the changes $a \rightarrow-a$ and (or) $z \rightarrow-z$, and hence it suffices to consider only the first quadrant $(a \geqslant 0, z \geqslant 0)$. In the first quadrant there exist four steady states

$$
O_{1}:(0,0), \quad O_{2}:(0,2), \quad O_{3}:\left(\frac{2}{\sqrt{3}}, 0\right), \quad O_{4}:\left(\sqrt{\frac{8}{15}}, \frac{2}{\sqrt{5}}\right) .
$$

The steady state at the origin $O_{1}$ is unstable, while the steady states on the axes $O_{2}$ and $O_{3}$ are stable, and $O_{4}$ is a saddle.

To get a complete picture of the phase space let us consider a set of nested elliptical curves

$$
W(a, z)=3 a^{2}+z^{2}=h .
$$

The derivative of $W(a, z)$ at $W=h$ along the solution curves is

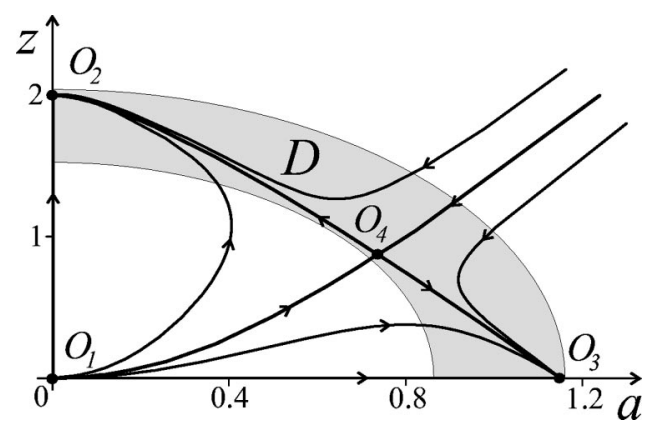

FIG. 15. Phase plane of the system Eq. (A3). 


$$
\frac{d W}{d t}=\varepsilon\left[z^{4}+z^{2}\left(1-\frac{5 h}{4}\right)-\frac{h^{2}}{4}+h\right] .
$$

Now we seek conditions on the parameter $h$ such that

$$
\left.\dot{W}\right|_{W=h}>0 \quad \text { or }\left.\quad \dot{W}\right|_{W=h}<0 \text { for } 0 \leqslant z \leqslant \sqrt{h} \text {. }
$$

From Eq. (A6) we determine that the derivative is negative for $h>4$ and is positive for $h<4(13+8 \sqrt{2}) / 41$. Consequently, in the phase plane there is an absorbing domain bounded by two nested elliptical curves

$$
D:\left\{(a, z) \mid \frac{4(13+8 \sqrt{2})}{41} \leqslant 3 a^{2}+z^{2} \leqslant 4\right\} .
$$

All trajectories enter into this domain and remain there. We note that $\mathrm{D}$ consists of two stable steady states $\mathrm{O}_{2}$ and $\mathrm{O}_{3}$, which are the only attractors for all trajectories. To confirm this we have to prove the absence of limit cycles inside $D$. To do this we use Bendixson's criterion [49]. The Bendixson function for Eq. (A3)

$$
F_{B}(a, z)=-\frac{3 \varepsilon}{8}\left(3 z^{2}+7 a^{2}-4\right)
$$

is negative in the domain $D$, and hence no closed orbits exist lying entirely in $D$. Thus the steady states $\mathrm{O}_{2}$ and $\mathrm{O}_{3}$ are the only attractors while the saddle $\mathrm{O}_{4}$ divides the phase plane (first quadrant) into the corresponding two basins of attraction (Fig. 15). Hence, in the original system Eq. (24) there exist three attractors: two steady states and a limit cycle in the plane $s=0$. For nonsmall $\varepsilon$, analogous results can be obtained for Eq. (24) by constructing a point map of the plane $x=0$ into itself. For the map we have two stable fixed points, one on the axis $r=0$ that corresponds to the steady state $(0,0, \sqrt{\mu})$ and another on the axis $s=0$, which corresponds to the limit cycle on the integral plane $s=0$, one unstable fixed point in the origin corresponding to the unstable steady state $(0,0,0)$, and a saddle corresponding to a hyperbolic limit cycle. The saddle divides the trajectories into two basins of attraction of the corresponding stable fixed points similar to Fig. 15.
[1] Yu. S. Kivshar and B. A. Malomed, Rev. Mod. Phys. 61, 763 (1989) (and references therein).

[2] T. Kawahara, Phys. Rev. Lett. 51, 381 (1983).

[3] S. Toh and T. Kawahara, J. Phys. Soc. Jpn. 54, 1257 (1985).

[4] T. Kawahara and S. Toh, Phys. Fluids 31, 2103 (1988).

[5] X.-L. Chu and M. G. Velarde, Phys. Rev. A 43, 1094 (1991).

[6] A. N. Garazo and M. G. Velarde, Phys. Fluids A 3, 2295 (1991).

[7] V. I. Nekorkin and M. G. Velarde, Int. J. Bifurcation Chaos Appl. Sci. Eng. 4, 1135 (1994).

[8] A. A. Nepomnyashchy and M. G. Velarde, Phys. Fluids 6, 187 (1994)

[9] C. I. Christov and M. G. Velarde, Physica D 86, 323 (1995).

[10] M. G. Velarde, V. I. Nekorkin, and A. G. Maksimov, Int. J. Bifurcation Chaos Appl. Sci. Eng. 5, 831 (1995).

[11] A. Ye. Rednikov, M. G. Velarde, Yu. S. Ryazantsev, A. A. Nepomnyashchy, and V. N. Kurdyumov, Acta Appl. Math. 39, 457 (1995).

[12] G. Huang, M. G. Velarde, and V. N. Kurdyumov, Phys. Rev. E 57, 5473 (1998).

[13] I. L. Kliakhandler, A. V. Porubov, and M. G. Velarde, Phys. Rev. E 62, 4959 (2000).

[14] B.-F. Feng and T. Kawahara, Physica D 139, 301 (2000).

[15] A. V. Porubov and M. G. Velarde, C. R. Acad. Sci., Ser. IIb: Mec., Phys., Chim., Astron. 328, 165 (2000).

[16] M. G. Velarde and A. Ye. Rednikov, in Nonlinear Waves in Multi-Phase Flow, edited by H.-C. Chang (Kluwer, Dodrecht, 2000), pp. 57-67.

[17] A. V. Porubov and M. G. Velarde, Wave Motion 31, 197 (2000).

[18] E. Picholle, C. Montes, C. Leycuras, O. Legrand, and J. Botineau, Phys. Rev. Lett. 66, 1454 (1991).

[19] C. Montes and A. M. Rubenchik, J. Opt. Soc. Am. B 9, 1857 (1992).
[20] P. D. Weidman, H. Linde, and M. G. Velarde, Phys. Fluids A 4, 921 (1992).

[21] H. Linde, X. Chu, and M. G. Velarde, Phys. Fluids A 5, 1068 (1993).

[22] H. Linde, X.-L. Chu, M. G. Velarde, and W. Waldhelm, Phys. Fluids A 5, 3162 (1993).

[23] M. G. Velarde, H. Linde, A. A. Nepomnyashchy, and W. Waldhelm, in Fluid Physics, edited by M. G. Velarde and C. I. Christov (World Scientific, London, 1995), pp. 433-441.

[24] V. I. Nekorkin, V. B. Kazantsev, N. F. Rulkov, and M. G. Velarde, IEEE Trans. Circuits Syst. 42, 785 (1995).

[25] H. Linde, M. G. Velarde, A. Wierschem, W. Waldhelm, K. Loeschcke, and A. Ye. Rednikov, J. Colloid Interface Sci. 188, 16 (1997).

[26] M. Santiago-Rosanne, M. Vignes-Adler, and M. G. Velarde, J. Colloid Interface Sci. 191, 65 (1997).

[27] A. Wierschem, M. G. Velarde, H. Linde, and W. Waldhelm, J. Colloid Interface Sci. 212, 365 (1999).

[28] A. Wierschem, H. Linde, and M. G. Velarde, Phys. Rev. E 62, $6522(2000)$.

[29] H. Linde, M. G. Velarde, W. Waldhelm, and A. Wierschem, J. Colloid Interface Sci. 236, 214 (2001).

[30] A. Wierschem, H. Linde, and M. G. Velarde, Phys. Rev. E (to be published).

[31] N. J. Zabusky and M. D. Kruskal, Phys. Rev. Lett. 15, 57 (1965).

[32] C. I. Christov, G. A. Maugin, and M. G. Velarde, Phys. Rev. E 54, 3621 (1996).

[33] V. A. Makarov, W. Ebeling, and M. G. Velarde, Int. J. Bifurcation Chaos Appl. Sci. Eng. 10, 1075 (2000).

[34] See e.g. M. Toda, Theory of Nonlinear Lattices (Springer, Berlin, 1981).

[35] R. Hirota and K. Suzuki, Proc. IEEE 61, 1483 (1973).

[36] A. C. Singer and A. V. Oppenheim, Int. J. Bifurcation Chaos Appl. Sci. Eng. 9, 571 (1999). 
[37] N. Islam, J. P. Singh, and K. Steiglitz, J. Appl. Phys. 62, 689 (1987).

[38] Y. Okada, S. Watanabe, and H. Tanaka, J. Phys. Soc. Jpn. 59, 2647 (1990).

[39] T. Kuusela and J. Hietarinta, Phys. Rev. Lett. 62, 700 (1989).

[40] Lord Rayleigh, The Theory of Sound (Dover, New York, 1945), Vol. 1, Chap. III, p. 81.

[41] I. Mitkov, Physica D 133, 398 (1999).

[42] P. Gray and S. K. Scott, Chemical Oscillations and Instabilities. Non-linear Chemical Kinetics (Clarendon, Oxford, 1994).

[43] J. D. Murray, Mathematical Biology (Springer, Berlin, 1993).
[44] H. Haken, Synergetics, 3rd ed. (Springer, Berlin, 1983).

[45] H. Haken, Advanced Synergetics (Springer, Berlin, 1983).

[46] B.-L. Adir, Semiconductors and Electronic Devices (Prentice Hall, Englewood Cliffs, NJ, 1984).

[47] M. W. Hirsch and S. Smale, Differential Equations, Dynamical Systems, and Linear Algebra (Academic, New York, 1974).

[48] W. Ebeling, U. Erdmann, J. Dunkel, and M. Jenssen, J. Stat. Phys. 101, 443 (2000).

[49] J. Guckenheimer and P. Holmes, Nonlinear Oscillations, Dynamical Systems and Bifurcations of Vector Fields (Springer, New York, 1983). 